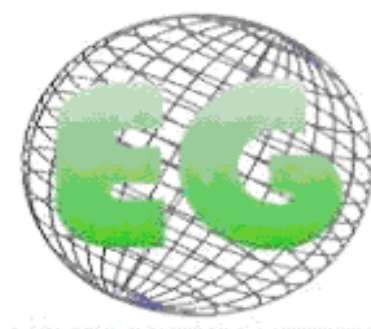

ISSN 1695.6141 $N^{\circ} 26$

\title{
Competencias del gestor hospitalario: estudio en un hospital público brasileño
}

Competências do gestor hospitalar: estudo em um hospital público brasileiro

\section{*Grohmann, MZ., *Battistella, LF., "*Baratto, JS.}

\begin{abstract}
*Profesoras del Departamento de Ciencias Administrativas de la Universidad Federal de Santa Maria. Email: marciazg@ufsm.br *Administradora y funcionaria del Hospital Universitario de la Universidad Federal de Santa Maria - Brasil.
\end{abstract}

Palabras clave: competencias; autoevaluación; importancia; carencias.

Palavras-chave: competências; auto avaliação; importância; carências.

Keywords: competences; self-assessment; importance; needs.

\section{RESUMEN}

El objetivo del estudio es conocer las competencias (conocimientos, habilidades y actitudes) del director del hospital, y sus objetivos son: identificar las competencias más importantes de acuerdo a los profesionales; medir las habilidades más desarrolladas en los profesionales encuestados, destacar las competencias con mayores carencias.

Se realizó un estudio descriptivo, utilizando el modelo de Lazarotto (2001) con 24 directivos de un hospital público brasileño. Los datos mostraron que, por lo general, las habilidades identificadas como las más importantes son también las más desarrolladas por los profesionales. Sin embargo, hay varias lagunas en las competencias de los directores de hospitales, siendo las principales en: políticas de salud pública, los sistemas de información, la planificación del desarrollo, la gestión estratégica.

Las habilidades que más necesitan ser desarrolladas son: dirigir el equipo de trabajo, ser agente de innovación y cambio, solucionar problemas y administrar los programas diseñados para motivar al equipo. Finalmente, las actitudes con mayores lagunas son: ser un líder educador, desarrollar la iniciativa y la autonomía del equipo y de los grupos comunitarios; ser creativo y estimular el trabajo en equipo.

En resumen, el trabajo es un primer análisis para identificar las lagunas en la formación de estos profesionales señalando que entre los tres tipos de competencias, el grupo de la Actitud es considerado el más importante por los profesionales y, por lo tanto, el que merece una mayor atención.

\section{RESUMO}

Este estudo teve como objetivo mapear as competências (conhecimentos, habilidades e atitudes) do gestor hospitalar, visando: identificar quais as competências mais importantes de acordo com os profissionais; medir quais as competências mais desenvolvidas nos profissionais pesquisados; levantar quais as competências com maiores carências. 
Para tanto, foi realizado um estudo descritivo, utilizando o modelo de Lazarotto (2001) com 24 gestores de um hospital público brasileiro. Os dados demonstraram que, normalmente, aquelas competências identificadas como mais importantes também são as que os profissionais mais desenvolveram. Porém, há várias lacunas nas competências dos gestores hospitalares, sendo que os principais conhecimentos são: políticas públicas de saúde; sistemas de informação; desenvolvimento de planejamento; administração estratégica.

As habilidades que mais precisam ser desenvolvidas são: gerenciar a equipe de trabalho; ser inovador e agente de mudanças; solucionar problemas; gerenciar os programas desenvolvidos e motivar a equipe de trabalho. Por fim, as atitudes com maiores lacunas são: ser um líder educador; desenvolver a iniciativa e a autonomia da equipe; incentivar a criatividade da equipe e dos grupos comunitários; ser criativo e estimular o trabalho em equipe.

Em suma, o trabalho serviu como primeira análise para identificação das lacunas na formação deste profissional e apontou que, dentre os três tipos de competências, o grupo da Atitude é considerado como o mais importante pelos profissionais e, portanto, o que merece uma maior atenção.

\section{ABSTRACT}

This study aimed to identify know hospital manager's' competencies (knowledge, skills and attitudes), so the objectives were: to identify the most important competences according to the professionals; measure what skills are most developed in these professionals, and find which competences need to be improved.

A descriptive study was made, using the Lazarotto model (2001) with 24 managers of a Brazilian public hospital. The data showed that, typically, those skills identified as most important are also the most developed - IT professionals. However, there are several gaps in the skills of hospital managers, and the main skills are: public health policies, information systems, development planning, strategic management.

The skills that need to be developed most are: work force management, to be an agent of innovatiion and change, troubleshooting, and management programs designed to motivate the team. Finally, the attitudes withthe biggest gaps are being a leading educator, developing initiative and autonomy of the team, encouraging the creativity of staff and community groups to be creative and encouraging teamwork.

In short, the work served as an initial analysis to identify gaps in the training of professionals and pointed out that among the three types of skills, attitude of the group is considered the most important by professionals and therefore deserves the most greater attention.

\section{INTRODUCCIÓN}

El hospital es una organización diferente, pues su existencia se debe a motivos no lucrativos cuyo enfoque es la atención al paciente y, por tanto, todas las acciones desarrolladas en la institución deben respetar la dignidad y los derechos de los pacientes.

Administrar este tipo de organización es algo extremadamente complejo que exige de los administradores conocimiento, habilidades y actitudes normalmente necesarias en cualquier gestor, pero también son necesarios aspectos distintos de la administración de las demás organizaciones.

La globalización, las transformaciones y el desarrollo en relación a los aspectos administrativos suceden de modo muy rápido y los cambios que ocurren en el mundo exigen innovaciones, renovación tecnológica, nuevos métodos de producción con menores costos, productos y servicios adecuados a las necesidades del ser humano. Estas transformaciones han exigido una preparación de los profesionales gerentes en salud con más competencias de las exigidas anteriormente. Detrás de esta agilidad en la toma de decisiones deben estar personas inteligentes y capaces de llevar a cabo este proceso con eficacia, ayudando a los 
hospitales y a los usuarios del Sistema Único de Salud a enfrentar las acciones en salud cada vez más exigentes.

El área de salud convive con la escasez de gerencia profesionalizada en gestión en sus varios niveless de actuación, principalmente en lo que respecta a la planificación, control e integración con el ambiente, por encontrarse tradicionalmente en manos de la clase médica $^{1,2}$.

Es decir, los profesionales que administran hospitales necesitan dominar contenidos del área de gestión, mas también conocer (en profundidad) aspectos específicos de atención a la salud. Por tanto, para tales profesionales no basta solo un curso formal de administración o un curso de enfermería o medicina, son necesarios ambos conocimientos.

Las organizaciones hospitalarias son consideradas organizacions complejas tanto con vistas a su estructura como a las relaciones sociales de trabajo ${ }^{3,4,5}$, lo que demanda de sus gestores competencias adecuadas a esa realidad.

Los profesionales que actuan en el sistema de salud son los responsables de la eficiencia del mismo, o sea, su capacidad de intervención es directamente proporcional a la calidad da su formación y/o capacitación para desarrollar actividades en los servicios de salud. Además de ser tambien prestador de cuidados, comparte junto con los otros profesionales la responsabilidad funcional de los servicios, a nivel asistencial, educativo y administrativo, siendo a veces un articulador del equipo.

Todos los servicios de salud dependen de las políticas públicas, de las condiciones de trabajo y de la capacidad gerencial para utilizar los recursos existentes, él es el responsable del desarrollo de este proceso. Axelrod et al. ${ }^{6}$ acreditan que "desarrollar y conservar talentos (colaboradores) será la mayor ventaja competitiva en el futuro", y vista la importancia estratégica de los gerentes, es imprescindible que las empresas se interesen en saber cuáles son las habilidades y competencias de los funcionarios que satisfacen sus expectativas. Pero ¿cómo identificar cuáles son éstas?

En este sentido, el presente estudio se justifica en la necesidad de obtener conocimiento sobre el proceso gerencial, que engloba el conocimiento, las habilidades y actitudes requeridas del gerente para el desarrollo gerencial de las organizaciones hospitalarias para transformar la realidad.

El trabajo se desarrolló en un Hospital Público vinculado a una Institución de Enseñanza Superior Brasileña (también conocido como Hospital Escuela) que se localiza en una ciudad del interior y es responsable de la atención a pacientes de diversos municipios de la región donde está localizada. Este hospital representa una de las pocas (y a veces única) posibilidades de atención gratuita que la población de la región posee.

En el sector administrativo del Hospital hay como regla, para el nombramiento de cargos de gerentes, la indicación de seguir un sesgo político, de acuerdo con las diferentes corrientes de pensamiento que están en la dirección. Esto significa que no siempre los profesionales escogidos poseen competencia suficiente para asumir tal cargo. Por ello, la imagen del Hospital depende de la eficiencia y de la excelencia de los servicios ofrecidos a la sociedad. Este cambio depende de personas con sus competencias, habilidades y actitudes, pudiendo transformar esa organización en una institución moderna, ágil y flexible con capacidad de innovar y empreender. 
Ante lo expuesto, el problema de investigación se definió como: ¿Los directores administrativos del Hospital poseen las competencias necesarias para el desempeño de sus actividades?

Los objetivos del estudio son: identificar las competencias (conocimientos, habilidades y actitudes) entendidas como ideales en el profesional gestor de salud; identificar el actual nivel de competencias de los profesionales del Hospital; identificar dónde se encuentran las mayores lagunas.

Atendiendo a la comprensión del problema, es necesario presentar un breve aporte teórico sobre competencias gerenciales. Para $\operatorname{Boog}^{7}$ (p.6), "competencia es la cualidad de quien es capaz de apreciar y resolver cierto asunto, hacer determinada cosa; significa capacidad, habilidad, aptitud e idoneidad". Durand ${ }^{8}$ (p.3) acredita que las competencias son "conjuntos de conocimientos, habilidades y actitudes interdependientes y necesarias para la consecución de un determinado propósito". De forma muy clara para Magalhães e Rocha ${ }^{9}$ (p.14) competencia es "un conjunto de conocimientos, habilidades y experiencias que acreditan a un profesional a ejercer determinada función".

En el contexto gerencial, el primer autor en trabajar con el concepto de competencias fue Boyatzis ${ }^{10}$ que lo utilizó para designar un conjunto de atributos que marcaban la formación de un perfil ideal para el gestor ${ }^{11}$.

El alcance de la investigación sobre competencias gerenciales es amplio y variado, reflejando la diversidad de enfoques que rodea al tema, incluyendo: desarrollo de competencias gerenciales ${ }^{12}$; competencias gerenciales como fuente de desempeño superior en las organizaciones ${ }^{13}$ y competencias gerenciales en diferentes contextos ${ }^{14}$.

Otro aspecto muy usual en términos de competencias gerenciales es su relación con conocimiento, habilidades y actitud que forman la tríada de la competencia gerencial (Figura 01).

Figura 1. Elementos Formadores de la Competencia

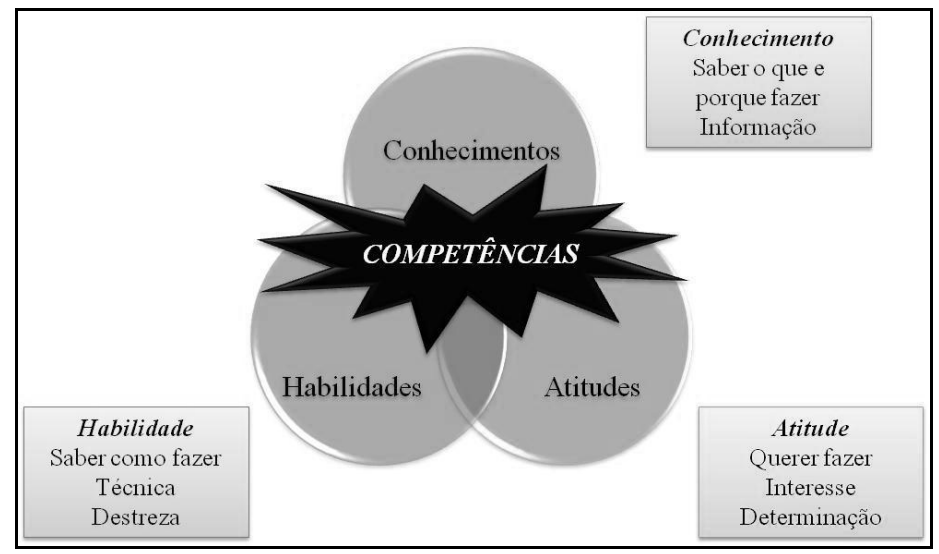

Fuente: adaptado de Durand 
Conforme se observa en el pensamiento de Wood e Picarelli ${ }^{15}$ para un buen profesional son necesarios un conjunto de habilidades, conocimiento y actitudes. Además de esto, los autores concluyen que el conocimiento incluye todas las técnicas e informaciones que el administrador domina y que son necesarias para el desempeño de su cargo. El principal conocimiento es la competencia técnica, que se puede decir que cubre la habilidad técnica, más la amplia realidad que ella consigue interpretar. Las actitudes forman la base de las opiniones según las cuales otras personas y los hechos, las ideas y los objetivos son vistos, interpretados y evaluados.

La unión de estos tres conceptos a la idea de competencia partió de Durand ${ }^{16}$ que construyó el concepto de competencia basado en tres dimensiones: conocimiento (información, saber qué, saber el porquê), habilidad (técnica, capacidad, saber como) y actitud (querer hacer, identidad, determinación).

Trabajando con la premisa anterior, Boterf ${ }^{17}$ postuló que estos conocimientos, habilidades y actitudes integradas en las competencias son, en realidad, recursos de competencias, siendo fundamentales para el desarrollo de las mismas y, como consecuencia, los tres aspectos deben ser trabajados en conjunto para la creación del conocimiento gerencial y mejora del desempeño gerencial.

\section{MÉTODO}

La investigación realizada se caracteriza por ser descriptiva, pues parte de un modelo teórico ya utilizado, por eso agrega al estudio la medición de la autoevaluación y no solo la importancia de los conocimientos, actitudes y habilidades que componen las competencias del gestor hospitalario. La figura 2 presenta todas las variables que miden cada uno de los constructos del modelo de Lazarotto ${ }^{18}$.

Los participantes de la investigación son profesionales, en su gran mayoría experimentados, con actuación profesional en gerencia hospitalaria. Del total de 31 gerentes existentes en el hospital, objeto de este estudio, participaron 24 profesionales.

El instrumento de recogida de datos utilizado fue el cuestionario. El mismo estaba dividido en dos etapas. En un primer momento, el cuestionario constaba de preguntas referentes al perfil personal y profesional de los entrevistados (conteniendo preguntas como: edad, estado civil, tiempo de actuación profesional, grado de instrucción, etc.). En un segundo momento, el instrumento presentaba el modelo propuesto por Lazarotto para medición de competencias gerenciales hospitalarias. Así, el instrumento presentaba 20 variables que medían conocimientos, 16 que medían habilidades y 15 que medían las actitudes que los profesionales consideraban tener (autoevaluación proesional). Destacar que las variables se presentaron de forma aleatoria en el cuestionario. De esa forma, el instrumento poseía 51 preguntas afirmativas, en las cuales, a través de una escala del tipo Likert, los encuestados debían posicionarse entre muy débil (1) excelente (5) para la medida de autoevaluación y entre (1) desacuerdo totalmente hasta (5) de acuerdo totalmente para medir el grado de importancia de las competencias del gestor hospitalario. 


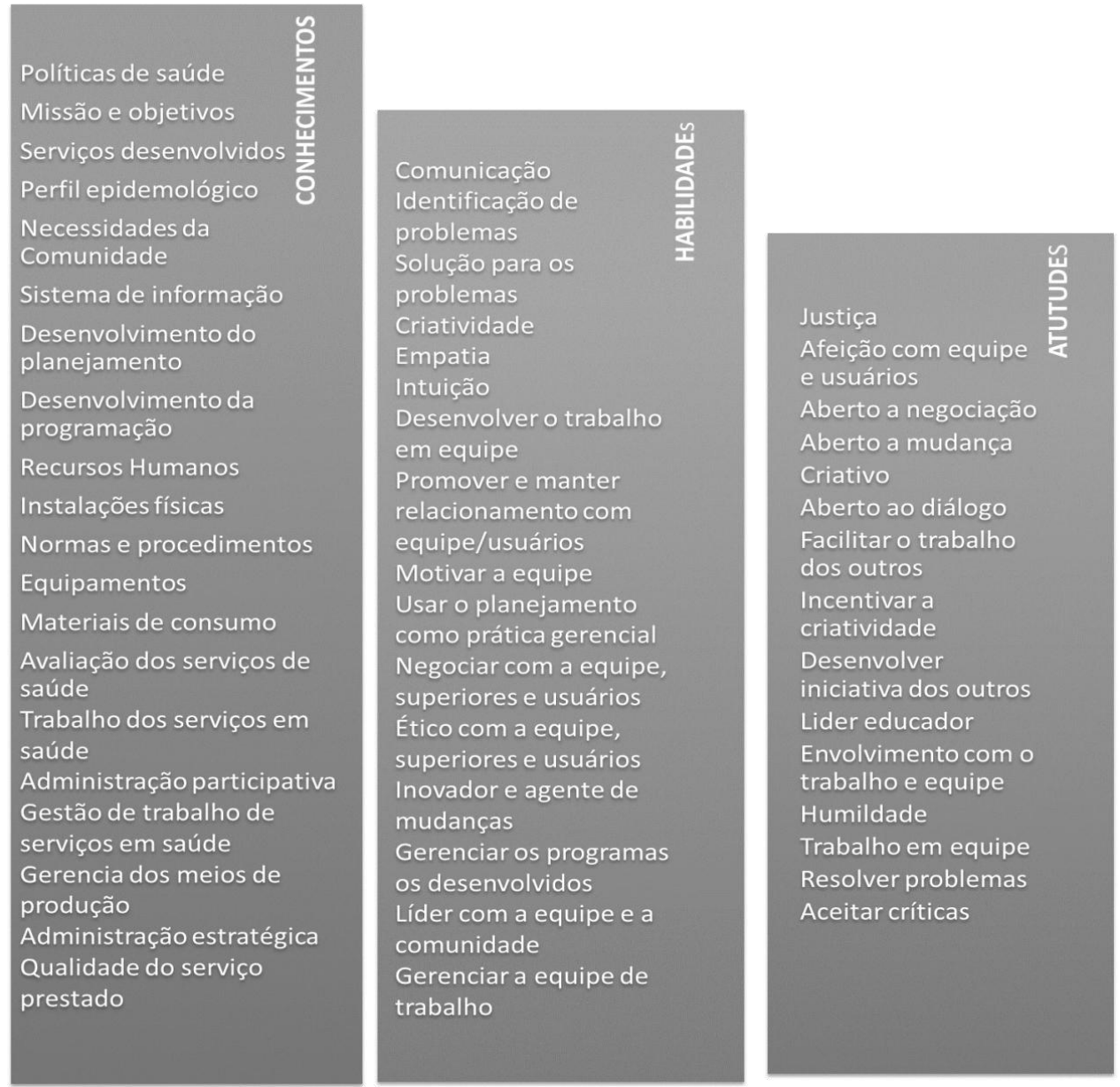

Fuente: elaborado por los autores, basado en Lazarotto

Los datos fueron tabulados y analizados estadísticamente a través del software SPSS. Tales análisis fueron elaborados de modo que pudiesen abarcar de manera correcta todas las cuestiones presentes en la investigación. Los resultados se analizaron de la siguiente manera:

- Perfil de los encuestados: uso de estadística descriptiva, a través de cálculos de frecuencia;

- Análisis de las competencias más importantes del gestor hospitalario: uso de estadística descriptiva, a través de cálculos de media y desvío- estándar;

- Análisis de la autoevaluación sobre las competencias del gestor hospitalario: uso de estadística descriptiva, a través de cálculos de média y desvío-estándar;

- Identificación de las principales lagunas en las competencias de los gestores del hospital: diferencia entre la media de la importancia atribuída y de la media de la autoevaluación para cada competencia del gestor hospitalario;

- Identificación del tipo de competencia con mayor importancia en el buen desempeño del gestor hospitalario: transformación de las variables que forman cada uno de los constructos (conocimientos, habilidades y actitudes) en una única media. 


\section{RESULTADOS}

Los resultados se presentan de la siguiente forma: se presenta la caracterización de la muestra; posteriormente se analiza cada uno de los constructos de la competencia, o sea, las informaciones sobre conocimientos, habilidades y actitud, realizándose tres análisis distintos: el grado de importancia, la autoevaluación y las principales carencias encontradas; por último, se presenta un panorama general de los constructos.

En relación a estos datos, se preguntó a las personas que trabajan en la parte administrativa del hospital sobre el género, edad, estado civil, grado de instrucción, experiencia profesional anterior, tiempo de trabajo en el hospital, cursos realizados (proporcionados por el hospital o no).

En lo que se refiere al género de los entrevistados, se observa que $45,8 \%$ son mujeres y $54,2 \%$ hombres. Las frajas de edad encontradas fueron: 26 a 35 años - 8,7\%; 36 a 40 años $-8,7 \%$; 41 a 45 años $-17,4 \%$; 46 a 50 años $-21,7 \%$; y más de 50 años $-43,5 \%$. Los datos demuestran que estos profesionales poseen franjas de edad más elevadas, es decir, son personas más maduras.

En cuanto a estado civil, la gran mayoría está casada $(53,8 \%)$ o separada $(23,1 \%)$, siendo que $11,5 \%$ están solteros. Por último, para completar las características personales de los encuestados, se observa que $8,37 \%$ tiene enseñanza media incompleta; $12,5 \%$ tiene enseñanza media completa; $25 \%$ superior incompleta; $37,5 \%$ pos-graduación completa y $16,7 \%$ pos-graduación incompleta, estos datos demuestran que la gran mayoría de los entrevistados tiene, como mínimo, curso superior completo.

Cuando se analizan los datos de la Tabla 01, referentes al tiempo de servicio prestado en el hospital, se observa que estamos tratando profesionales, en su mayoría, experimentados. Esta información es importante para el objetivo de este estudio que es el de trazar las competencias necesarias a los profesionales responsables de la administración de hospitales, dado que personas con poca experiencia en el cargo tal vez sintiesen dificultad para detectar qué conocimientos, habilidades y actitudes son necesarias para el buen desempeño profesional.

\begin{tabular}{lcc}
\multicolumn{4}{c}{ Tabla 01- Tiempo de actuación en el hospital } \\
\hline & Frecuencia & Porcentaje \\
\hline Menos de 5 años & 2 & 08,7 \\
6 a 10 años & 2 & 08,7 \\
11 a 15 años & 5 & 21,7 \\
16 a 20 años & 4 & 17,4 \\
21 a 25 años & 4 & 17,4 \\
Más de 25 años & 6 & 26,1 \\
\hline Total & 24 & 100 \\
\hline
\end{tabular}

En lo que se refiere a las informaciones profesionales, se observa que $95,7 \%$ de los respondientes realizaron algún tipo de curso promovido por el propio hospital, de ayuda al desempeño profesional. Además de este alto porcentaje, otro hecho que corrobora la continua busca de mejora de estos profesionales es que $83,3 \%$ también procuraron hacer otros cursos, buscando el perfeccionamiento profesional. 
En suma, la caracterización del perfil de los encuestados muestra que estos son, en su gran mayoría, personas maduras, con curso superior, que buscan continuamente el crecimiento profesional y que poseen tiempo de actuación en el hospital, es decir, con gran experiencia profesional. Se trata de personas altamente capacitadas para ayudar a responder al problema y objetivos de la presente investigación.

Conforme mencionado anteriormente, este estudio tuvo por base el modelo de Lazarotto que trabaja con 20 variables para medir conocimientos, 16 para habilidades y 15 para actitudes. Así, los próximos resultados seguirán tal división.

Las tablas de 02 a 04 indican las medias de los factores relacionados con cada uno de los constructos, presentando el grado de acuerdo o desacuerdo de los encuestados en relación a las frases del cuestionario. Las tablas también apuntan el grado de importancia de los gestores hospitalarios para cada uno de los conocimientos, actitudes y habilidades listados en el instrumento de medida. Cabe resaltar que cuanto más próximo a 5 es el valor de la media de la variable, mayor fue la autoevaluación del gerente, dado que 5 es la opción "excelente evaluación".

Como es posible observar en la Tabla 02, las medias encontradas para las variables del constructo conocimiento tuvieron una variación entre 4,04 a 2,83. En cuanto a la desviación estándar, el valor giró en torno de 1,00.

Los conocimientos que presentaron mayores medias fueron, respectivamente: misión y objetivos $(4,04)$, servicios desarrollados $(3,88)$, perfil epidemiológico $(3,83)$ normas y procedimientos $(3,75)$, instalaciones físicas $(3,59)$ y equipamientos $(3,50)$. Todas estas variables presentaron medias superiores a 3,50, lo que demuestra que los gerentes tienen un buen conocimiento sobre la marcha de las actividades internas de la organización hospitalaria.

En el otro extremo, las variables que presentaron menores medias fueron: materiales de consumo (2,96); gerencia de los medios de producción (2,92); evaluación de los servicios de salud $(2,88)$; gestión del trabajo en servicios de salud $(2,83)$, y necesidades de la comunidad $(2,83)$. Además, todas las medias encontradas en el constructo conocimientos se encuentran por encima de 2,50. Como la escala utilizada variaba de 1 a 5 , se puede considerar que cualquier valor por encima de 2,50 es satisfactorio.

Tabla 02- Conocimientos del Gestor Hospitalario

\begin{tabular}{l|c|c|c|c|c}
\hline \multicolumn{1}{c}{ Conocimientos } & \multicolumn{2}{c|}{$\begin{array}{c}\text { Auto- } \\
\text { evaluación }\end{array}$} & \multicolumn{2}{c|}{ Importancia } & \multirow{2}{*}{ Iif. } \\
\cline { 2 - 5 } & media & desvío & media & desvío & \\
\hline C01 Políticas de salud & 3,17 & 0,916 & 4,21 & 0,832 & 1,04 \\
C02 Misión y objetivos & 4,04 & 0,806 & 4,54 & 0,658 & 0,50 \\
C03 Servicios desarrollados & 3,88 & 0,806 & 4,54 & 0,658 & 0,70 \\
C04 Perfil epidemológico & 3,83 & 0,916 & 4,46 & 0,721 & 0,63 \\
C05 Necesidades de la Comunidad & 2,83 & 0,816 & 3,63 & 0,875 & 0,81 \\
C06 Sistema de información & 3,25 & 0,846 & 4,25 & 0,896 & 1,00 \\
C07 Desarrollo de la planificación & 3,13 & 0,991 & 4,13 & 0,899 & 1,00 \\
C08 Desarrollo de la programación & 3,00 & 0,780 & 3,88 & 0,797 & 0,88 \\
C09 Recursos Humanos & 3,21 & 0,832 & 3,96 & 0,858 & 0,75
\end{tabular}


C10 Instalaciones físicas

C11 Normas y procedimientos

C12 Equipamientos

C13 Materiales de consumo

C14 Evaluación de los servicios de salud

\begin{tabular}{l|l|l|l|l}
3,58 & 1,017 & 4,17 & 0,916 & 0,59 \\
3,75 & 0,737 & 4,29 & 0,858 & 0,54 \\
3,50 & 0,884 & 3,88 & 1,034 & 0,38 \\
2,96 & 1,122 & 3,50 & 1,021 & 0,54 \\
2,88 & 0,797 & 3,75 & 0,944 & 0,87 \\
3,00 & 0,722 & 3,65 & 0,934 & 0,65 \\
3,13 & 0,740 & 3,96 & 0,750 & 0,83 \\
2,83 & 0,816 & 3,63 & 0,875 & 0,80 \\
& & & & \\
2,92 & 1,138 & 3,83 & 0,816 & 0,91 \\
& & & & \\
3,04 & 1,065 & 4,00 & 0,852 & 0,96 \\
3,33 & 0,868 & 4,17 & 0,916 & 0,84
\end{tabular}

De forma general, las principales carencias encontradas por los gerentes son especifícamente respecto a los límites del hospital (se resalta que la variable C04 - perfil epidemiológico - escapa a esta regla).

Tras la identificación de los conocimientos que los gestores hospitalarios tienen, se sigue con el levantamiento de los conocimientos juzgados como de mayor importancia para el buen desempeño profesional. En este sentido, se observa en la Figura 3 que tres de los conocimientos clasificados en las primeras posiciones se refieren a cuestiones relacionadas con el tratamiento directo al paciente: servicios desarrollados (primer lugar); perfil epidemiológico (tercer lugar) y normas y procedimientos (cuarto lugar). En el otro extremo, como conocimientos menos importantes aparecen tres factores directamente ligados a las políticas públicas de salud: trabajo de los servicios en salud y gestión de trabajo de servicio de salud (ambos en decimoséptimo lugar) y necesidades de la comunidad (decimonoveno lugar). Por otro lado, los conocimientos específicos del área gerencial se encuentran clasificados en las posiciones intermedias en importancia.

Figura 3 - Jerarquía de importancia de los conocimientos del Gestor Hospitalario

\begin{tabular}{|l|l|}
\hline Classificação & Conhecimentos \\
\hline $01^{\circ}$ & Serviços desenvolvidos \\
$02^{\circ}$ & Missão e objetivos \\
$03^{\circ}$ & Perfil epidemiológico \\
$04^{\circ}$ & Normas e procedimentos \\
$05^{\circ}$ & Sistema de aviso \\
$06^{\circ}$ & Políticas de saúde \\
$07^{\circ}$ & Qualidade do serviço prestado \\
$07^{\circ}$ & Instalações físicas \\
$09^{\circ}$ & Desenvolvimento do planejamento \\
$10^{\circ}$ & Administração estratégica \\
$11^{\circ}$ & Administração participativa \\
$11^{\circ}$ & Recursos humanos \\
$13^{\circ}$ & Desenvolvimento da programação \\
$13^{\circ}$ & Equipamentos \\
$15^{\circ}$ & Gerencia dos meios de produção \\
$16^{\circ}$ & Avaliação dos serviços de saúde \\
$17^{\circ}$ & Trabalho dos serviços de saúde \\
$17^{\circ}$ & Gestão de trabalho de serviços em saúde \\
$19^{\circ}$ & Necessidades da comunidade \\
$20^{\circ}$ & Materiais de consumo \\
\hline
\end{tabular}


De esta forma, se constata que los gestores hospitalarios señalan que los conocimientos más importantes son aquellos más inmediatistas y urgentes y que el trabajo de este profesional es tan complejo que las funciones secundarias como gerenciamiento proactivo y de conocimiento de políticas públicas son cuestiones "secundarias" o no tan relevantes para el buen desempeño profesional.

Finalmente, el último análisis sobre los conocimientos del gestor hospitalario trata sobre las principales carencias encontradas en el hospital objeto de este estudio. Estos resultados son consecuencia de la diferencia encontrada entre la importancia atribuida a cada conocimiento y la autoevaluación de los encuestados (última columna de la Tabla 2).

Los datos señalan que la secuencia encontrada, las principales lagunas en los conocimientos y, por tanto, los puntos en los cuales los gestores necesitan de mejora son: políticas públicas de salud (primer lugar); sistemas de información y desarrollo de planificación (ambos en segundo lugar); administración estratégica (cuarto lugar); gerencia de los medios de producción (quinto lugar); desarrollo de la programación (sexto lugar); evaluación de los servicios de salud (séptimo lugar); calidad del servicio prestado (octavo lugar); administración participativa (noveno lugar); necesidades de la comunidad (décimo lugar); gestión de trabajo de servicios en salud (decimoprimer lugar); recursos humanos (decimosegundo lugar); servicios desarrollados (decimotercer lugar); trabajo de los servicios en salud (decimocuarto lugar); perfil epidemiológico (decimoquinto lugar); instalaciones físicas (decimosexto lugar); normas y procedimientos y materiales de consumo (ambos en decimoséptimo lugar); misión y objetivos (decimonoveno lugar) y equipamientos (vigésimo lugar)

Se observa que entre los primeros lugares aparecen varios conocimientos própios del área de administración y, de esta forma, una de las sugerencias para la mejora de la gestión en el hospital sería la realización de cursos de actualización sobre administración.

La Tabla 03 muestra las medias encontradas para las 16 variables del constructo habilidades. La variación encontrada fue de 4,29 a 3,50, con desvío-estándar cerca de 0,80.

Tabla 03- Habilidades del Gestor Hospitalario

\begin{tabular}{l|c|c|c|c|c}
\hline \multirow{2}{*}{\multicolumn{1}{c|}{ Habilidades }} & \multicolumn{2}{|c|}{$\begin{array}{c}\text { Auto- } \\
\text { evaluación }\end{array}$} & \multicolumn{2}{c|}{ Importancia } & \multirow{2}{*}{ Dif. } \\
\cline { 2 - 5 } & media & desvío & media & desvío & \\
\hline H01 Comunicación & 3,92 & 0,717 & 4,67 & 0,701 & 0,75 \\
H02 Identificación de problemas & 3,83 & 0,637 & 4,67 & 0,481 & 0,84 \\
H03 Solución de los problemas & 3,71 & 0,690 & 4,67 & 0,564 & 0,96 \\
H04 Creatividad & 3,96 & 0,750 & 4,71 & 0,464 & 0,75 \\
H05 Empatía & 3,79 & 0,721 & 4,58 & 0,583 & 0,79 \\
H06 Intuición en el desarrollo del trabajo & 3,83 & 0,761 & 4,25 & 0,737 & 0,42 \\
H07 Desarrollar el trabajo en equipo & 3,92 & 0,775 & 4,75 & 0,607 & 0,83 \\
H08 Promover y manter relación con & 4,00 & 0,722 & 4,79 & 0,508 & 0,79 \\
$\quad$ equipo/usuarios & & & & & \\
H09 Motivar al equipo & 3,71 & 0,860 & 4,63 & 0,710 & 0,92 \\
H10 Usar la planificación como prática & 3,58 & 0,829 & 4,42 & 0,717 & 0,84 \\
$\quad$ gerencial & & & & &
\end{tabular}


H11 Negociar con el equipo, superiores y usuarios

H12 Ético con el equipo, superiores y usuarios

H13 Innovador y agente de cambios

H14 Gerenciar los programas desarrollados

H15 Líder con el equipo y la comunidad H16 Gerenciar el equipo de trabajo

\begin{tabular}{l|l|l|l|l}
3,79 & 0,883 & 4,46 & 0,721 & 0,67 \\
4,29 & 0,690 & 4,71 & 0,624 & 0,42 \\
& & & & \\
3,50 & 0,780 & 4,46 & 0,721 & 0,96 \\
3,54 & 0,931 & 4,46 & 0,658 & 0,92 \\
& & & & \\
3,75 & 0,846 & 4,50 & 0,722 & 0,75 \\
3,75 & 0,989 & 4,83 & 0,481 & 1,08
\end{tabular}

Las habilidades consideradas por los gerentes como puntos fuertes de su desempeño fueron: ser ético $(4,29)$; promover y manter relacioness $(4,00)$; creatividad $(3,96)$; desarrollar trabajo en equipo $(3,92)$, y comunicación $(3,92)$. Por otro lado, las menores medias encontradas fueron: encontrar solución para problemas $(3,71)$; motivar al equipo $(3,71)$; usar la planificación como práctica gerencial $(3,58)$; dirigir programas $(3,54)$, y ser innovador o agente de cambios $(3,50)$.

Todas las 16 variables sobre habilidades presentaron medias superiores a 3,50, lo que demuestra que los gerentes tienen sus habilidades profesionales muy bien desarrolladas. Destaca que en el constructo anterior (conocimientos), 15 variables presentaron medias inferiores a 3,50.

Los resultados indican que las tres habilidades más importantes apuntadas por los gestores hospitalarios están directamente relacionadas con el trabajo en equipo: gerenciar el equipo de trabajo (primero lugar); promover y mantener relación con equipo y usuarios (segundo lugar) y desarrollar el trabajo en equipo (tercer lugar). Como habilidades menos importantes aparecen: usar la planificación como práctica gerencial (decimoquinto lugar) e intuición en el desarrollo del trabajo (decimosexto lugar).

Se observa (Figura 4) que a pesar de que las tres primeras habilidades están relacionadas, las habilidades de relación y las habilidades técnicas se entrecruzan. Por ello, en las seis primeras ubicaciones aparecen solo habilidades de relación, hecho que indica que en un servicio con alto nivel de estrés y que trabaja con vidas humanas, las cuestiones interpersonales asumen una gran importancia, superando las cuestiones técnicas.

Figura 4 - Jerarquía de importancia de las habilidades del Gestor Hospitalario

\begin{tabular}{|l|l|}
\hline \multicolumn{1}{|c|}{ Classificação } & Habilidades \\
\hline $01^{\circ}$ & Gerenciar a equipe de trabalho \\
$02^{\circ}$ & Promover e manter relacionamento com equipe/usuários \\
$03^{\circ}$ & Desenvolver o trabalho de equipe \\
$04^{\circ}$ & Criatividade \\
$04^{\circ}$ & Ético com a equipe, superiores e usuários \\
$06^{\circ}$ & Comunicação \\
$06^{\circ}$ & Identificação de problemas \\
$06^{\circ}$ & Solução de problemas \\
$09^{\circ}$ & Motivar a equipe \\
$10^{\circ}$ & Empatia \\
$11^{\circ}$ & Líder com a equipe e a comunidade \\
$12^{\circ}$ & Gerenciar os programas desenvolvidos \\
$12^{\circ}$ & Inovador e agente de mudanças \\
$12^{\circ}$ & Negociar com a equipe, superiores e usuários \\
$15^{\circ}$ & Usar o planejamento como prática gerencial \\
$16^{\circ}$ & Intuição no desenvolvimento do trabalho \\
\hline
\end{tabular}


El último análisis sobre las habilidades del gestor hospitalario se refiere a las principales carencias encontradas en el hospital, apuntadas por la diferencia encontrada y presentada en la última columna de la Tabla 3.

Los resultados muestran que las habilidades que precisan ser más desarrolladas son: dirigir el equipo de trabajo (primer lugar); ser innovador y agente de cambios y solucionar problemas (ambos en segundo lugar); gerenciar los programas desarrollados y motivar al equipo (ambos en cuarto lugar); usar la planificación como práctica gerencial e identificar problemas (ambos en sexto lugar); desarrollar el trabajo en equipo (octavo lugar); empatía y promover y manter relacionamento con equipo y usuarios (ambos en noveno lugar); comunicación, creatividad y lidiar con el equipo o usuarios (todos en decimoprimer lugar); negociar con el equipo, superiores y usuarios (decimocuarto lugar); y, por último, intuición en el desarrollo del trabajo y ser ético con el equipo, superiores y usuarios (ambos en decimoquinto lugar).

\section{Tabla 4 - Actitudes del Gestor Hospitalario}

\begin{tabular}{l|c|c|c|c|c}
\hline \multicolumn{1}{c|}{ Actitudes } & \multicolumn{2}{c|}{$\begin{array}{c}\text { Auto- } \\
\text { evaluación }\end{array}$} & Importancia & Dif. \\
\cline { 2 - 4 } & media & desvío & media & desvío & \\
\hline A01 Ser justo con su equipo y usuarios & 4,44 & 0,598 & 4,87 & 0,344 & 0,43 \\
A02 Ser afectivo, dando atención al & 4,29 & 0,690 & 4,67 & 0,564 & 0,38 \\
$\quad$ equipo y usuarios & & & & & \\
A03 Estar abierto a la negociación & 4,25 & 0,737 & 4,54 & 0,658 & 0,29 \\
A04 Estar abierto a los cambios & 4,21 & 0,721 & 4,67 & 0,637 & 0,46 \\
A05 Ser creativo y estimular la creatividad & 3,83 & 0,701 & 4,58 & 0,583 & 0,75 \\
$\quad$ del equipo & & & & & \\
A06 Estar abierto al diálogo y saber & 4,33 & 0,637 & 4,75 & 0,607 & 0,42 \\
$\quad$ escuchar & & & & & \\
A07 Ser facilitador del trabajo con el & 3,88 & 0,612 & 4,42 & 0,653 & 0,54 \\
$\quad$ equipo y grupos de la comunidad & & & & & \\
A08 Incentivar la creatividad del equipo y & 3,63 & 0,824 & 4,38 & 0,710 & 0,75 \\
$\quad$ de los grupos comunitarios & & & & & \\
A09 Desarrollar la iniciativa y la autonomía & 3,67 & 0,868 & 4,42 & 0,653 & 0,75 \\
$\quad$ del equipo y de los grupos & & & & & \\
A10 Ser un líder educador & 3,54 & 0,977 & 4,54 & 0,721 & 1,00 \\
A11 Tener participación con el trabajo, con & 4,08 & 0,974 & 4,75 & 0,531 & 0,67 \\
$\quad$ el equipo y comunidad & & & & & \\
A12 Demostrar humildad & 4,04 & 0,721 & 4,71 & 0,624 & 0,50 \\
A13 Privilegiar el trabajo en equipo & 4,21 & 0,954 & 4,54 & 0,977 & 0,50 \\
A14 Saber resolver problemas & 3,92 & 0,717 & 4,63 & 0,646 & 0,71 \\
A15 Saber aceptar críticas & 3,83 & 0,816 & 4,54 & 0,883 & 0,71 \\
\hline
\end{tabular}

El constructo actitudes (Tabla 4) fue el que presentó mayor amplitud en las medias ya que las mismas oscilaron entre 4,43 y 3,54. Así, comparando las menores medias con los otros dos constructos, se observa que las medias de actitud fueron las más elevadas (menor media conocimiento $=2,83$; menor media habilidad $=3,50$ ). 
La actitud más desarrollada entre los gerentes investigados fue la de ser justo con su equipo y usuarios $(4,43)$, y en la secuencia aparecen: estar abierto al diálogo y saber escuchar $(4,33)$; ser afectivo, prestando atención al equipo y usuarios $(4,29)$; estar abierto a la negociación (4,25); estar abierto a los cambios $(4,21)$; favorecer el trabajo en equipo $(4,21)$; tener participación con el trabajo, con el equipo y comunidad $(4,08)$, y mostrar humildad $(4,04)$. Todas estas variables presentaron medias superiores a 4,00 , lo que demuestra que las actitudes son el punto fuerte de los gerentes entrevistados.

Tras la presentación de la autoevaluación de las actitudes de los gestores del hospital, se pasa a la identificación del grado de importancia de las mismas. En este sentido, se observa en la Figura 5 que las cinco actitudes más importantes se refieren a relaciones de trabajo, principalmente con los colegas: ser justo con su equipo y usuarios (primer lugar); estar abierto al diálogo y saber escuchar y tener participación con el trabajo, con el equipo y con la comunidad (ambos en segundo lugar); favorecer el trabajo en equipo (cuarto lugar) y ser afectivo, dando atención al equipo y usuarios (quinto lugar).

\section{Figura 5 - Jerarquía de importancia de las actitudes del Gestor Hospitalario}

\begin{tabular}{|l|l|}
\hline Classificação & Atitudes \\
\hline $01^{\circ}$ & Ser justo com sua equipe e usuários \\
$02^{\circ}$ & Ser aberto ao diálogo e saber escutar \\
$02^{\circ}$ & Ter envolvimento com o trabalho, equipe e comunidade \\
$04^{\circ}$ & Privilegiar o trabalho em equipe \\
$05^{\circ}$ & Ser afetivo, dando atenção para equipe e usuários \\
$05^{\circ}$ & Ser aberto às mudanças \\
$07^{\circ}$ & Saber resolver problemas \\
$08^{\circ}$ & Ser criativo e estimular a criatividade da equipe \\
$09^{\circ}$ & Ser aberto à negociação \\
$09^{\circ}$ & Ser um líder educador \\
$09^{\circ}$ & Demonstrar humildade \\
$09^{\circ}$ & Saber aceitar críticas \\
$13^{\circ}$ & Ser facilitador do trabalho com equipe e grupos da comunidade \\
$14^{\circ}$ & Desenvolver a iniciativa e a autonomia da equipe e dos grupos \\
$15^{\circ}$ & Incentivar a criatividade da equipe e dos grupos comunitários \\
\hline
\end{tabular}

Las actitudes consideradas como de menor importancia también se refieren al trabajo en equipo, por ello se relacionan con cuestiones ligadas al incentivo de la mejora del trabajo en el equipo: ser facilitador del trabajo con el equipo y grupos de la comunidad (decimotercer lugar); desarrollar la iniciativa y la autonomía del equipo y de los grupos (decimocuarto lugar) e incentivar la creatividad del equipo y de los grupos comunitarios (decimoquinto lugar).

Finalmente, el último análisis sobre las actitudes del gestor hospitalario se refiere a las principales carencias encontradas en el hospital, objeto de este estudio. Estos resultados se derivan de la diferencia encontrada entre la importancia atribuida a cada conocimiento y la autoevaluación de los encuestados (última columna de la Tabla 4).

Los datos indican que la secuencia encontrada, o sea, las principales lagunas en las actitudes y, por tanto, los puntos en los cuales los gestores necesitan de mejora son: ser un líder educador (primer lugar); desarrollar la iniciativa y la autonomía del equipo, incentivar la creatividad del equipo y de los grupos comunitarios y ser creativo y estimular el trabajo en equipo (todos en segundo lugar); saber resolver problemas y saber aceptar críticas (ambos en quinto lugar); tener participación con el trabajo, con el equipo y con la comunidad (séptimo lugar); ser facilitador del trabajo con el equipo y con los grupos de la comunidad (octavo lugar); mostrar humildad y favorecer el trabajo en equipo (ambos en noveno lugar); 
estar abierto a los cambios (décimo primer lugar); ser justo con el equipo y usuarios (decimosegundo lugar); estar abierto al diálogo y saber escuchar (decimotercer lugar); ser afectivo, prestando atención al equipo y usuarios (decimocuarto lugar); y, por último, estar abierto a la negociación (decimoquinto lugar).

Para completar la presentación de los resultados la Tabla 5 muestra las medias obtenidas de los conocimientos, actitudes y habilidades que forman las competencias del gestor hospitalario. Para ello, el primero paso fue la transformación de cada uno de los componentes de la competencia gerencial en una única variable. El procedimiento adoptado en esa etapa, fue el de la creación de una nueva variable, resultante del cálculo de las medias de cada variable que formaba el constructo. Tal información es el resultado de la media de las medias de las variables que median cada uno de los tres constructos.

Tabla 5- Medias de los constructos que forman las competencias del Gestor Hospitalario

\begin{tabular}{l|c|c|c|c|c}
\hline \multirow{2}{*}{ Factor } & \multicolumn{2}{|c|}{ Auto-evaluación } & \multicolumn{2}{c|}{ Importancia } & \multirow{2}{*}{ Dif. } \\
\cline { 2 - 5 } & media & desvío & media & desvío & \\
\hline Conocimiento & 3,26 & 0,604 & 4,02 & 0,608 & 0,76 \\
Habilidad & 3,81 & 0,598 & 4,60 & 0,440 & 0,79 \\
Actitudes & 4,05 & 0,584 & 4,66 & 0,442 & 0,62 \\
\hline
\end{tabular}

Como se puede observar, el constructo conocimiento obtuvo una media final, en términos de autoevaluación de los entrevistados, de 3,26 (en una escala de 1 a 5), el constructo habilidades obtuvo 3,80 de media y el constructo actitudes 4,05 de media. De esta forma, se verifica que los profesionales responsables der la administración del hospital objeto de este estudio consideran que su mejor desempeño está en actitudes, seguido de habilidades. Y las principales carencias se encuentran en lo tocante a conocimientos.

Respecto a la importancia de cada constructo, los datos muestran que los gestores hospitalarios consideran como aspecto más importante de sus competencias las habilidades (media de 4,66), en la secuencia aparecen las habilidades (media de 4,60) y, por último, los conocimientos (media de 4,02).

Para completar, las principais carencias, según los gestores entrevistados están, respectivamente, en las habilidades (diferencia entre las medias de 0,79), en los conocimientos (diferencia entre las medias de 0,76) y la menor carencia encontrada en el hospital público analizado fue en las actitudes (diferencia entre las medias de 0,62).

\section{DISCUSIONES}

El trabajo de los gestores de salud es extremadamente complejo $^{19}$ y necesita de competencias técnicas y humanas. Diversos estudios, como los de Benavides, Moya, Segura, Porta y Amela; Gómez, Turrell ${ }^{20}$ Melo, Costa, Fávero, Trevizan, Hayashida ${ }^{21}$ buscaron identificar cuáles serían las competencias ideales para estos profesionales y otros como los de Santos y Castro ${ }^{22}$ Huerta-Riveros, Lyton-Pavez e Saldia-Barahona ${ }^{23}$; Furukawa, Cunha e $\mathrm{Kowal}^{24}$ analizaron cuáles son las competencias actuales de los gestores de salud.

Estos trabajos señalaron diversas semejanzas con el presente estudio, siendo la más importante la de que para los profesionales de gestión de salud las competencias humanas son, normalmente, más importantes que las técnicas. La importancia del trabajo en equipo 
fue destacada en el estudio de Queiroz e Araújo ${ }^{25}$ e Martins $^{26}$, el liderazgo en los de Moniz, Cavalcanti y Araújo ${ }^{27}$ y Santos y Bittencourt ${ }^{28}$

Algunos resultados que, en un primer momento, pueden no tener relación directa con el trabajo de los gestores de salud y que fueron encontrados en este estudio, son corroborados por otros estudios. En este sentido, se cita la importancia de las estrategias ${ }^{29}$ de la gestión de la calidad ${ }^{30}$, los sistemas de informaciones ${ }^{31}$ y la necesidad de creatividad e innovación ${ }^{32}$

Un aspecto apuntado por otros estudios y que merece destacarse es el hecho de que los gestores hospitalarios no pueden (o deben) ser meros enfermeros. Se justifica tal afirmativa por el hecho de que, para dirigir unidades de salud son necesarias competencias gerenciales, que no siempre son desarrolladas en las universidades de enfermería ${ }^{33,34}$. Otro motivo son los constantes conflictos entre médicos y enfermeros, derivados de peleas por el poder $^{35,36}$.

\section{CONCLUSIONES}

El presente estudio trató de identificar cuál de los constructos que componen las competencias gerenciales (conocimientos, habilidades y actitudes) posee mayor carencia en los profesionales de un hospital público. Para ello fue necesario identificar cuál es el nivel de importancia atribuido a varios conocimientos, habilidades y actitud; cuál es la autoevaluación que los profesionales realizaron sobre sus conocimientos, habilidades y actitudes y, por último, a través de la comparación, identificar las principales lagunas.

La investigación partió de un modelo teórico ya utilizado (Lazzarotto, 2001) y contribuyó para el medio académico dado que todavía existen pocos artículos publicados en los principales periódicos y eventos nacionales de nivel $A$ que aborden esa temática. Los resultados pueden ayudar a investigaciones futuras y contribuir en el medio empresarial con la intención de mejorar el desempeño de los gerentes hospitalarios.

El primer objetivo del estudio fue el de identificar las competencias (conocimientos, habilidades y actitudes) entendidas como ideales al profesional gestor de salud. En este sentido los resultados señalaron que los conocimientos clasificados en las primeras posiciones se refieren a cuestiones relacionadas con el tratamiento directo al paciente: servicios desarrollados (primer lugar); perfil epidemiológico (tercer lugar) y normas y procedimientos (cuarto lugar). Las habilidades más importantes para este profesional están directamente relacionadas con el trabajo en equipo y son: dirigir el equipo de trabajo; promover y mantener relaciones con el equipo y usuarios y desarrollar el trabajo en equipo. Y las actitudes más importantes se refieren a relaciones de trabajo, principalmente con los colegas: ser justo con su equipo y usuarios; estar abierto al diálogo y saber escuchar y tener participación con el trabajo, con el equipo y con la comunidad; favorecer el trabajo en equipo y ser afectivo, prestando atención al equipo y usuarios.

El segundo objetivo del estudio era identificar el actual nivel de competencias de los profesionales del Hospital. Los conocimientos que presentaron mayores medias fueron, respectivamente: misión y objetivos; servicios desarrollados; perfil epidemiológico; normas y procedimientos; instalaciones físicas y equipos. Las habilidades consideradas por los gerentes como puntos fuertes de su desempeño fueron: ser ético; promover y mantener relaciones; creatividad; desarrollar trabajo en equipo y comunicación. La actitud más desarrollada entre los gerentes encuestados fue la de ser justo con su equipo y usuarios, y en la secuencia aparecen: estar abierto al diálogo y saber escuchar; ser afectivo, atender al equipo y usuarios; estar abierto a la negociación; estar abierto a los cambios; favorecer el 
trabajo en equipo; tener participación con el trabajo, con el equipo y comunidad y mostrar humildad.

El último objetivo del estudio era el de identificar donde se encuentran las mayores lagunas en las competencias de los gestores hospitalarios encuestados. Las principales lagunas en los conocimientos son: políticas públicas de salud; sistemas de información y desarrollo de planificación; administración estratégica. Las habilidades que precisan desarrollarse más son: dirigir el equipo de trabajo; ser innovador y agente de cambios y solucionar problemas; gerenciar los programas desarrollados y motivar al equipo; usar la planificación como práctica gerencial e identificar problemas; desarrollar el trabajo en equipo. Y las principales lagunas en las actitudes son: ser un líder educador; desarrollar la iniciativa y la autonomía del equipo; incentivar la creatividad del equipo y de los grupos comunitarios; ser creativo; estimular el trabajo en equipo; saber resolver problemas, y saber aceptar críticas.

Como limitaciones del estudio, se puede citar el hecho de que el tema competencias es un tema que está en evidencia y aún carece de investigaciones empíricas, como el enfoque de la temática del presente artículo. Otro factor limitante lo constituye la imposibilidad de conseguir recolectar los datos de todos los gerentes que trabajan en el hospital. Para investigaciones futuras, se sugiere investigar en otros segmentos como comercio o industria con la intención de conferir una propuesta más concreta sobre la interacción entre competencias gerenciales y desempeño profesional.

\section{REFERENCIAS}

1. Alves M, Penna, CMM, Brito, MJM. Perfil dos gerentes de unidades básicas de saúde. Rev Bras Enferm, Brasília (DF). 2004 jul-ago; 57 (4): 441-6.

2. Keller A, Burlamaqui P. Organizações hospitalares e corpo clínico: gerenciando custos ou saúde? Anais do XXVII - Encontro Nacional da Associação dos Programas de PósGraduação em Administração. Curitiba, 2004.

3. Lima-Gonçalves E. Estrutura organizacional do hospital moderno. RAE - Revista de Administração de Empresas. 1998; 38 (1): 80-90.

4. Lima-Gonçalves $\mathrm{E}$. Condicionantes internos e externos da atividade do hospital-empresa. ERA - eletrônica; 2004; 1 (2).

5. Feuerwerker LCM, Cecílio LCO. O hospital e a formação em saúde: desafios atuais. Ciência \& Saúde Coletiva. 2007; 12 (4): 965-971.

6. Machado $\mathrm{MH}$. Gestão do trabalho em saúde no contexto de mudanças. Rev Ad Public 2000; 34: 136-46.

7. Boog G. O desafio da competência. São Paulo, Editora Best Seller, 1991.

8. Durand T. Strategizing for innovation: competence analysis in assessing strategic change. In: Competence-based strategic management. Sanchez R, Heene A (Ed.). Chichester, England: Wiley, 1997.

9. Magalhães S, Rocha M. (1997). Desenvolvimento de Competências: o futuro agora! Rev. Treinamento Desenvolvimento. 1997, pp.12- 14.

10. Boyatzis RE. The competent management: a model for effective performance. New York: John Wiley, 1982.

11. Wood R, Payne T. Competency based Recruitment and Selection - a Practice Guide. London, Wiley, 1998

12. Wester D. Developing managerial competence, developing corporate competence. Human Resource Manag. Jour. 1998; 8(2):145-167. 
13. Abraham SE, Karns LA, Shaw K, Mena MA. Managerial competencies and the managerial performance appraisal process. Jour. Manag. Development. 2001; 20 (10): 842852.

14. Anderson P, Pulich M. Managerial competencies necessary in today's dynamic health care environment. Health Care Manager, Wisconsin, 2002.

15. Wood TJ, Picarelli VF. Remuneração e carreira por habilidades e por competências: preparando a organização para a era das empresas de conhecimento intensivo. São Paulo: Atlas, 2004

16. Durand $T$ (1998). Forms of incompetence. Fourth International Conference on Competence Based Management. Oslo: Norwegian School of Management, 1988.

17 Boterf GL. (1997). Construire la Competence Collective de Lémtreprise. Gestion, 1997; 22 (3).

18. Lazzarotto EM. Competências essenciais requeridas para o gerenciamento das unidades básicas de saúde. Mestrado em Engenharia de Produção Universidade Federal de Santa Catarina, UFSC, Brasil, 2001.

19. Benavides FG, Moya C, Segura A, Puente ML, Porta M, Amela C. Las competencias profesionales en Salud Pública. Gac Sanit. 2006 Jun; 20 (3): 239-243.

20. Gómez DT, Tyrrell MA. La enfermera: conocimientos y competencias para dirigir hospitales. Esc. Anna Nery. 2010 Mar; 14(1): 71-75.

21. Melo MR, Costa A, Fávero N, Trevizan MA, Hayashida M. Expectativa do administrador hospitalar frente as funções administrativas realizadas pelo enfermeiro. Rev. Latino-Am. Enfermagem . 1996 Jan ; 4(1): 131-144.

22. Santos I, Castro CB. Características pessoais e profissionais de enfermeiros com funções administrativas atuantes em um hospital universitário. Rev. esc. enferm. USP, 2010 Mar; 44(1): 154-160.

23. Huerta-Riveros PC, Leyton-Pavez CE, Saldia-Barahona H. Análisis de las competencias directivas de una red de salud pública. Rev. salud pública. 2009 Dec; 11(6): 979-987.

24. Furukawa PO, Cunha IC, Kowal O. Profile and competencies of nurse managers at accredited hospitals. Rev. Latino-Am. Enfermagem. 2011 Feb ; 19(1): 106-114.

25. Queiroz EH, Araujo TCF. Trabalho em Equipe: Um Estudo Multimetodológico em Instituição Hospitalar de Reabilitação. Interamerican Journal of Psychology, 2007; 41221-230 26. Martins JJ. Humanização nas práticas de saúde: o discurso coletivo dos trabalhadores, gestores e usuários de um estabelecimento assistencial de saúde hospitalar (EASH). Interface - Comunicação, Saúde, Educação2009; 13.

27. Moniz AF, Cavalcanti L, Araújo TCF. Voluntariado hospitalar: um estudo sobre a percepção dos profissionais de saúde. Estudos de Psicologia, 2008, 13 (Agosto).

28. Santos Id, Bittencourt Castro C. Estilos e dimensões da liderança: iniciativa e investigação no cotidiano do trabalho de enfermagem hospitalar. Texto \& Contexto Enfermagem. 2008; 17734-742

29. Gonçalves MA, Indelicato ZJ, Amorim AC. Gestão estratégica hospitalar: aplicação de custos na saúde. Revista de Administração Faces Journal; 2009; 8161-179.

30. Vieira MM, Gurgel GD. Qualidade total e administração hospitalar: explorando disjunções conceituais. Ciência e Saúde Coletiva2002; 7325-334.

31. Xavier JF, Adriano AL. A Tecnologia da Informação na Área Hospitalar: um Caso de Implementação de um Sistema de Registro de Pacientes. RAC. Revista de Administração Contemporânea. 2001; 5105-120.

32. Feldman LB, Ruthes RM, Cunha IC, Kowal O Criatividade e inovação: competências na gestão de enfermagem. Rev. bras. enferm. 2008 Apr.

33. Lourenção DCA, Benito G, Véles A. Competências gerenciais na formação do enfermeiro. Rev. bras. enferm. 2010 Feb; 63(1): 91-97. 
34. Cavalcanti SC, Yoshikawa EE, Barros S. Constituição de competências a serem desenvolvidas para a intervenção dos processos saúde: doença da população no ensino de terceiro grau em enfermagem. Rev. esc. enferm. USP. 2001 Mar; 35(1): 95-95

35. Corrêa LMF, Coutinho FG, Vieira A. Relações De Poder E Decisão: Conflitos Entre Médicos E Administradores Hospitalares. Revista De Administração Mackenzie. 2010; 1131 54.

36. Gómez DT, Leite JI, Moura M. Conflicto Generado Por La Designación De Enfermeras Para La Dirección Hospitalaria - Una Visión Periodística. Escola Anna Nery Revista De Enfermagem. 2008; 12102-107. 